\title{
The Landau-Placzek Ratio for Multicomponent Fluids
}

\author{
H. N. W. LEKKERKERKER $\dagger$ and W. G. LAIDLAW $\$$ \\ Faculté des Sciences \\ Université Libre de Bruxelles \\ 1050 Brussels, Belgium
}

Received June 1, 1972

\begin{abstract}
Under the assumption that the coupling between the sound modes and modes associated with heat and mass diffusion can be neglected, an expression for the Landau-Placzek ratio for multicomponent fluids is derived using thermodynamic fluctuation theory. Applications of the general formula to ternary systems are discussed briefly.
\end{abstract}

\section{Introduction}

The spectrum of light scattered from a dense fluid consists of three lines: the central Rayleigh line and the Brillouin doublet the components of which are shifted symmetrically about the frequency of the incident light. Landau and Placzek ${ }^{(1)}$ showed that for a onecomponent fluid the ratio of the integrated intensity of the Rayleigh line, $I_{\mathrm{R}}$, to that of the sum of the shifted lines, $2 I_{\mathrm{B}}$, is given by

$$
\frac{I_{\mathrm{R}}}{2 I_{\mathrm{B}}}=\frac{c_{p}-c_{v}}{c_{v}}=\gamma-1
$$

where $c_{p}$ and $c_{v}$ are the specific heats at constant pressure and constant volume respectively. The recent literature ${ }^{(2,3,4)}$ contains a number of expressions for the Landau-Placzek ratio, $I_{\mathrm{R}} / 2 I_{\mathrm{B}}$, for binary mixtures, obtained under the assumption that the coupling between the sound modes and modes associated with heat and mass diffusion can be neglected. From results obtained for binary mixtures ${ }^{(5,6)}$ it appears that light scattering spectroscopy could become a valuable tool in solution thermodynamies and the formu-

$\uparrow$ NATO Science Fellow 1971-1972.

‡ On leave from the Chemistry Department, University of Calgary, Calgary, Alberta, Canada. 
lation of the Landau-Placzek ratio for multicomponent fluids therefore seems appropriate.

Jordan and Jordan $\dagger^{(2)}$ and Mountain and Deutch $\star^{(4)}$ obtain their results from their calculation of the spectral intensity distribution for binary mixtures. However, for the purpose of obtaining just the Landau-Placzek ratio for multicomponent fluids, the extension of spectrum calculations is not indispensable. Indeed under the assumption of decoupling of the pressure fluctuations from the entropy and concentration fluctuations, Miller ${ }^{(3)}$ obtained the Landau-Placzek ratio for a binary mixture from thermodynamic fluctuation theory only.

In this paper the use of thermodynamic fluctuation theory to obtain the Landau-Placzek ratio is extended to systems with an arbitrary number of components. The present approach differs from Miller's treatment in the choice of the state variables with attendant simplications in the thermodynamic derivations.

\section{The Landau-Placzek Ratio for Multicomponent Fluids}

The temperature dependence of the optical dielectric constant at constant density and composition is neglected, so that the fluctuation in the optical dielectric constant, $\delta \epsilon$, is given by

$$
\delta \epsilon=\left(\frac{\partial \epsilon}{\partial \rho}\right)_{T,\{c\}} \delta \rho+\sum_{j=1}^{n-1}\left(\frac{\partial \epsilon}{\partial c_{j}}\right)_{T, \rho,\left\{c^{\prime}\right\}} \delta c_{j}
$$

The subscript $\{c\}$ denotes the $n-1$ independent mass fractions, $c_{1}, c_{2}, \ldots c_{n-1}$, and the prime in $\left\{c^{\prime}\right\}$ indicates that the mass fraction appearing in the partial derivative is omitted from the set $\{c\}$. To make effective use of the decoupling of the sound modes and modes associated with heat and mass diffusion one should write $\delta \epsilon$ in terms of the pressure fluctuation, $\delta p$, and of the fluctuations in a set of variables which are statistically independent of $\delta p$. From thermodynamic fluctuation theory ${ }^{(7)}$ it follows that $\delta s, \delta c_{1}, \delta c_{2}, \ldots \delta c_{n-1}$ is

$\uparrow$ In order to apply the expression given by Jordan and Jordan (Ref. 2, Eqs. 2-73) one must remove one of the thermodynamic variables kept constant in the partial derivatives appearing in this equation. Specifically whenever $P$ or $V$ occur as subscripts in the partial derivatives they should be omitted.

‡ In the denominator of the expression given by Mountain and Deutch ( ${ }^{(4)}$, Eq. 4.1) the quantity $(\partial \epsilon / \partial p)_{s, c}$ should appear instead of $(\partial \epsilon / \partial p)_{T, c}$. 
such a set. Further, to facilitate separation of the contribution of the concentration fluctuations to the Landau-Placzek ratio, it is advantageous to use instead of $\delta s$ the fluctuation

$$
\delta s_{\text {red }}=\delta s-\sum_{j=1}^{n-1}\left(\frac{\partial s}{\partial c_{j}}\right)_{T, p,\left\{c^{\prime}\right\}} \delta c_{j}
$$

which is statistically independent of the concentration fluctuations. $\dagger$ The quantity $\delta s_{\text {red }}$ will be referred to as the reduced entropy fluctuation because of the analogy of its definition with that of the reduced heat flow, ${ }^{(11)}$ extensively used in irreversible thermodynamics. Starting from Eq. (1) and making use of Eq. (2) the fluctuation in the optical dielectric constant can be rewritten as

$$
\delta \epsilon=\left(\frac{\partial \epsilon}{\partial \rho}\right)_{T,\{c\}}\left(\frac{\partial \rho}{\partial s}\right)_{p,\{c\}} \delta s_{\mathrm{red}}+\left(\frac{\partial \epsilon}{\partial \rho}\right)_{T_{,\{c\}}}\left(\frac{\partial \rho}{\partial p}\right)_{s,\{c\}} \delta p+\sum_{j=1}^{n-1}\left(\frac{\partial \epsilon}{\partial c_{j}}\right)_{T, p,\left\{c^{\prime}\right\}} \delta c_{j}
$$

Here the Rayleigh line is due to entropy and concentration fluctuations and the Brillouin lines are due to pressure fluctuations, so that making use of Eq. (3) one can immediately write

$$
\begin{aligned}
& \frac{I_{\mathrm{R}}}{2 I_{\mathrm{B}}}= \\
& \frac{\left(\frac{\partial \epsilon}{\partial \rho}\right)_{T,\{c\}}^{2}\left(\frac{\partial \rho}{\partial s}\right)_{p,\{c\}}^{2}\left\langle\left(\delta s_{\mathrm{red}}\right)^{2}\right\rangle+\sum_{j, k=1}^{n-1}\left(\frac{\partial \epsilon}{\partial c_{j}}\right)_{T, p,\left\{c^{\prime}\right\}}\left(\frac{\partial \epsilon}{\partial c_{k}}\right)_{T, p,\left\{c^{\prime}\right\}}\left\langle\delta c_{j} \delta c_{k}\right\rangle}{\left(\frac{\partial \epsilon}{\partial \rho}\right)_{T,\{c\}}^{2}\left(\frac{\partial \rho}{\partial p}\right)_{s\{c\}}^{2}\left\langle(\delta p)^{2}\right\rangle}
\end{aligned}
$$

where the brackets $\langle\ldots\rangle$ indicate the average over an equilibrium ensemble. Considering the fluctuations in a volume $V^{*}$ which is a small portion of a macroscopically large system and using thermodynamic fluctuation theory ${ }^{(7)}$ one obtains, for the mean square fluctuations

$$
\begin{aligned}
\left\langle\left(\delta s_{\text {red }}\right)^{2}\right\rangle & =\frac{k_{\mathrm{B}} c_{p}}{\rho V^{*}} \\
\left\langle(\delta p)^{2}\right\rangle & =\frac{k_{\mathrm{B}} T}{V^{*} X_{s}} \\
\left\langle\delta c_{j} \delta c_{k}\right\rangle & =\frac{k_{\mathrm{B}} T^{\top}\left(\mathbf{B}^{-1}\right)_{j k}}{\rho V^{*}}
\end{aligned}
$$

$\dagger$ Similar variables have been used by Cohen et al. ${ }^{(8)}$ and Prigogine and coworkers ${ }^{(9,10)}$.

PACOL A 2 
Here $k_{\mathrm{B}}$ is Boltzmann's constant, $X_{s}$ is the adiabatic compressibility and the matrix $\mathbf{B}^{-1}$ is the inverse of the symmetric matrix $\mathbf{B}$, whose elements are defined as

$$
B_{j k}=\left(\frac{\partial\left[\mu_{j}-\mu_{n}\right]}{\partial c_{k}}\right)_{T, p,\left\{c^{\prime}\right\}} \equiv\left(\frac{\partial \tilde{\mu}_{j}}{\partial c_{k}}\right)_{T, p,\left\{c^{\prime}\right\}}
$$

Substitution of Eq. (5) in (4) and using standard thermodynamic relations ${ }^{(7)}$ allows $I_{\mathrm{R}} / 2 I_{\mathrm{B}}$ to be rewritten as

$$
\frac{I_{\mathrm{R}}}{2 I_{\mathrm{B}}}=(\gamma-1)+\frac{c_{0}{ }^{2} \sum_{j, k=1}^{n-1}\left(\frac{\partial \epsilon}{\partial c_{j}}\right)_{T, p,\left\{c^{\prime}\right\}}\left(\frac{\partial \epsilon}{\partial c_{k}}\right)_{T, p,\left\{c^{\prime}\right\}}\left(\mathbf{B}^{-1}\right)_{j k}}{\rho^{2}\left(\frac{\partial \epsilon}{\partial \rho}\right)_{T,\{c\}}^{2}}
$$

where $c_{0}$ is the low-frequency sound velocity. This is an exact expression for the Landau-Placzek ratio for multicomponent systems within the framework of the decoupling assumption and the neglect of $(\partial \epsilon / \partial T)_{\rho,\{c\}}$. Note that since $\mathbf{B}^{-1}$ is a positive definite matrix for a stable thermodynamic system the second term on the right-hand side of Eq. (7) is always positive.

\section{Applications}

From the general expression for the Landau-Placzek ratio one can immediately recover the result for the binary case by setting $n=2$. Since all terms in Eq. (7) are well defined quantities, one can treat, directly, a multicomponent fluid by setting $n$ equal to the appropriate value and formulating explicitly the elements of $\mathbf{B}$. For example, for a three component ideal solution one obtains after some manipulations

$$
\begin{aligned}
& \mathrm{B}_{11}=\frac{R T}{D c_{1} c_{3}}\left\{c_{1} c_{2} M_{1}+\left(1-c_{2}\right)^{2} M_{2}+c_{2} c_{3} M_{3}\right\} \\
& \mathrm{B}_{12}=\mathrm{B}_{21}=\frac{R T}{D c_{3}}\left\{\left(1-c_{1}\right) M_{1}+\left(1-c_{2}\right) M_{2}-c_{3} M_{3}\right\} \\
& \mathrm{B}_{22}=\frac{R T}{D c_{2} c_{3}}\left\{\left(1-c_{1}\right)^{2} M_{1}+c_{1} c_{2} M_{2}+c_{1} c_{3} M_{3}\right\}
\end{aligned}
$$

where $D=c_{1} M_{2} M_{3}+c_{2} M_{1} M_{3}+c_{3} M_{1} M_{2}$ and the $M_{i}$ 's and $c_{i}$ 's are the molecular weights and equilibrium mass fractions respectively. With these quantities the elements of the inverse matrix $\mathbf{B}^{-1}$ may be obtained and the result inserted directly into Eq. (7). 
For purposes of analysis it may be advantageous to introduce statistically independent concentration variables so that the contribution of the concentration fluctuations to the Landau-Placzek ratio ean be obtained directly as a sum of squares. In a three component system it can easily be shown that the fluctuations in the variable $c_{1, \text { red, }}$ which is defined as

$$
c_{1, \text { red }}=c_{1}-\left(\frac{\partial c_{1}}{\partial c_{2}}\right)_{p, T, \tilde{\mu}_{1}} c_{2}
$$

are statistically independent of the fluctuations in the remaining concentration variable $c_{2}$. Using this new variable the LandauPlaczek ratio for a three component system can be written as

$$
\frac{I_{\mathrm{R}}}{2 I_{\mathrm{B}}}=(\gamma-1)+\frac{c_{0}{ }^{2}\left\{\left(\frac{\partial \epsilon}{\partial c_{1}}\right)_{p, T, c_{2}}^{2}\left(\frac{\partial \tilde{\mu}_{1}}{\partial c_{1}}\right)_{p, T, c_{2}}^{-1}+\left(\frac{\partial \epsilon}{\partial c_{2}}\right)_{p, T, \tilde{\mu}_{1}}^{2}\left(\frac{\partial \tilde{\mu}_{2}}{\partial c_{2}}\right)_{p, T, \tilde{\mu}_{1}}^{-1}\right\}}{\rho^{2}\left(\frac{\partial \epsilon}{\partial \rho}\right)_{T,\{c\}}^{2}}
$$

where

$$
\begin{aligned}
& \left(\frac{\partial \epsilon}{\partial c_{2}}\right)_{p, T, \tilde{\mu}_{1}}=\left(\frac{\partial \epsilon}{\partial c_{2}}\right)_{p, T, c_{1}}+\left(\frac{\partial \epsilon}{\partial c_{1}}\right)_{p, T, c_{2}}\left(\frac{\partial c_{1}}{\partial c_{2}}\right)_{p, T, \tilde{\mu}_{1}} \\
& \left(\frac{\partial \tilde{\mu}_{2}}{\partial c_{2}}\right)_{p, T, \tilde{\mu}_{1}}=\left(\frac{\partial \tilde{\mu}_{2}}{\partial c_{2}}\right)_{p, T, c_{1}}+\left(\frac{\partial \tilde{\mu}_{2}}{\partial c_{1}}\right)_{p, T, c_{2}}\left(\frac{\partial c_{1}}{\partial c_{2}}\right)_{p, T, \tilde{\mu}_{1}}
\end{aligned}
$$

The decomposition of the concentration fluctuation contribution to the Landau-Placzek ratio, displayed by Eq. (10) can be used to advantage. For example, it is clear from Eq. (12) that when $c_{2}$ is small (i.e. an impurity) $\left(\partial \tilde{\mu}_{2} / \partial c_{2}\right)_{p, T, \tilde{\mu}_{1}}$ is large, with the result that only concentration fluctuations of component 1 contribute significantly to the second term on the r.h.s. of Eq. (10). As another example where it is convenient to use Eq. (10), consider the case of a dilute ternary solution. Taking component 3 as solvent it can easily be shown that $\left(\partial c_{1} / \partial c_{2}\right)_{p, T, \tilde{\mu}_{1}}$ is small and thus

$$
\frac{I_{\mathrm{R}}}{2 I_{\mathrm{B}}} \simeq(\gamma-1)+\frac{c_{0}{ }^{2}\left\{\left(\frac{\partial \epsilon}{\partial c_{1}}\right)_{p, T, c_{2}}^{2}\left(\frac{\partial \tilde{\mu}_{1}}{\partial c_{1}}\right)_{p, T, c_{2}}^{-1}+\left(\frac{\partial \epsilon}{\partial c_{2}}\right)_{p, T, c_{1}}^{2}\left(\frac{\partial \tilde{\mu}_{2}}{\partial c_{2}}\right)_{p, T, c_{1}}^{-1}\right\}}{\rho^{2}\left(\frac{\partial \epsilon}{\partial \rho}\right)_{T,\{c\}}^{2}}
$$

so that the concentration fluctuation contribution to the LandauPlaczek ratio is the sum of two binary mixture-like terms. 
For fluids with three or more components it appears to be difficult to make more general statements on the Landau-Placzek ratio, than those made above. For example the fact that the second term in Eq. (11) could be opposite in sign to the first along with the variability of the remaining terms in Eq. (10) makes it difficult to predict trends in the Landau-Placzek ratio, even for ternary systems.

\section{Acknowledgements}

The authors wish to acknowledge the hospitality extended by Prof. Prigogine's group and the constructive criticisms of Dr. J. P. Boon. The award of a NATO Science Fellowship by the Netherlands Organization for the Advancement of Pure Research to HNWL and the sabbatical leave granted to WGL by the University of Calgary are acknowledged

\section{REFERENCES}

1. See Frenkel, J., Kinetic Theory of Liquids, Dover Publications, Ine,, New York, 1955, p. 244.

2. Jordan, P. C. and Jordan, J. P., J. Chem. Phys. 45, 2492 (1966).

3. Miller, G. A., J. Phys. Chem. 41, 2305 (1967).

4. Mountain, R. D. and Deutch, J. M., J. Chem. Phys. 50, 1103 (1969).

5. Miller, G. A. and Lee, C. S., J. Phys. Chem. 72, 4644 (1968).

6. Dubois, M. and Berge, P., Phys. Rev. Letters 26, 121 (1971).

7. Landau, L. D. and Lifshitz, E. M., Statistical Physics, Pergamon Press, London, 1958, Chapter 12.

8. Cohen, C., Sutherland, J. W. H. and Deutch, J. M., Phys. Chem. Liquids 2, 213 (1971).

9. Glansdorff, P. and Prigogine, I., Thermodynamic Theory of Structure, Stability and Fluctuations, Wiley-Interscience, London, 1971, Chapter 2.

10. Sanfeld, A. and Steinchen-Sanfeld, A., Bull. Cl. Sc. Acad. Roy. Belg. 5, LVII, 684 (1971).

11. Meixner, J. and Reik, H. G., "Thermodynamik der Irreversible Prozessen ", in Handbuch der Physik, Vol. III(2), ed. S. Flügge, Springer, Berlin, 1959 . 